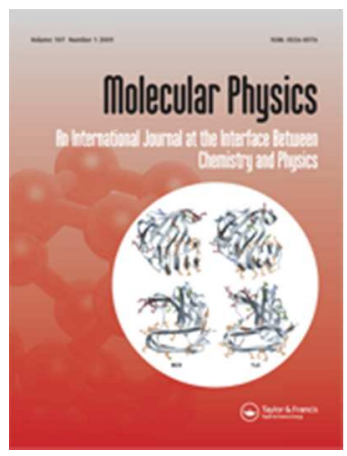

\title{
Light absorption and excitation energy transfer calculations in primitive photosynthetic bacteria
}

\begin{tabular}{|r|l|}
\hline Journal: & Molecular Physics \\
\hline Manuscript ID: & Draft \\
\hline Manuscript Type: & Full Paper \\
\hline Complete List of Authors: & $\begin{array}{l}\text { Komatsu, Yu; University of Tsukuba, ; University of Tsukuba, Graduate } \\
\text { School of Pure and Applied Sciences } \\
\text { Kayanuma, Megumi; University of Tsukuba, } \\
\text { Shoji, Mitsuo; University of Tsukuba, Graduate School of Pure and Applied } \\
\text { Sciences } \\
\text { Yabana, Kazuhiro; University of Tsukuba, Graduate School of Pure and } \\
\text { Applied Sciences }\end{array}$ \\
\hline Keywords: & $\begin{array}{l}\text { photosynthesis, quantum chemistry, excitation energy transfer, extrasolar } \\
\text { planets }\end{array}$ \\
\hline \multicolumn{2}{|c|}{} \\
\hline
\end{tabular}

\section{SCHOLARONE ${ }^{\text {M }}$ \\ Manuscripts}




\title{
Light absorption and excitation energy transfer calculations in primitive photosynthetic bacteria
}

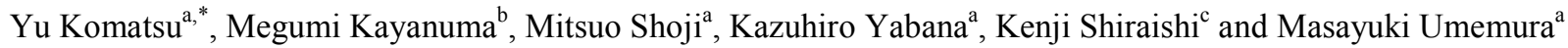 \\ ${ }^{\mathrm{a}}$ Graduate School of Pure and Applied Sciences, University of Tsukuba, Tsukuba, Japan \\ ${ }^{\mathrm{b}}$ Graduate School of Systems and Information Engineering, University of Tsukuba, Tsukuba, Japan \\ ${ }^{\mathrm{c}}$ Graduate School of Engineering, Nagoya University, Nagoya, Japan
}

\begin{abstract}
In photosynthetic organisms, light energy is converted into chemical energy through the light absorption and excitation energy transfer (EET) processes. These processes start in light-harvesting complexes, which contain special photosynthetic pigments. The exploration of unique mechanisms in light-harvesting complexes is directly related to studies, such as artificial photosynthesis or biosignatures in astrobiology. We examined, through ab initio calculations, the light absorption and EET processes using cluster models of light-harvesting complexes in purple bacteria (LH2). We evaluated absorption spectra and energy transfer rates using the LH2 monomer and dimer models to reproduce experimental results. After the calibration tests, a LH2 aggregation model, composed of 7 or 19 LH2s aligned in triangle lattice, was examined. We found that the light absorption is red-shifted and the energy transfer becomes faster as the system size increases. We also found that EET is accelerated by exchanging the central pigments to lower-energy excited pigments. As an astrobiological application, we calculated light absorptions efficiencies of the LH2 in different photoenvironments.
\end{abstract}

Keywords: photosynthesis; quantum chemistry; excitation energy transfer; extrasolar planets

*Corresponding author. Email: yukomatu@ccs.tsukuba.ac.jp 


\section{Introduction}

For photosynthetic organisms on earth, light energy from the Sun is the main energy source, which is converted into their vital chemical energy. Elucidating of the energy conversion mechanisms attracted a great amount of attention in different scientific fields, with applications closely related to current topics including the development of artificial photosynthesis and production of novel clean energies. In the first step of photosynthesis in light-harvesting complexes (LHCs), light absorption occurs. Then, excitation energy transfers (EET) are accomplished remarkably efficiently by collective electronic excitations across photosynthetic pigments, and the excitation energy is trapped in a reaction centre (RC), where subsequent charge separations and chemical reactions take place. In these light-harvesting processes, photosynthetic organisms utilize either or both of two types of LHCs: the peripheral antenna complexes, and the core antenna complexes. Purple bacteria possess light-harvesting complex II (LH2) and reaction center-light-harvesting complex I (RC-LH1), which correspond to the peripheral and core antenna complexes, respectively, and are one of the simplest antenna systems. Excitation energy collected by the LH2 transfers to the RC-LH1. The LH2 has been studied extensively since its crystal structure was solved in 1995 (1). The LH2 has a circular structure and contains two rings composed of bacteriochlorophylls $a$ (Bchls $a$ ), one of the photosynthetic pigments (2) (Figure 1). The inner ring is called B850 and has an absorption maximum around 850 $\mathrm{nm}$, and the outer ring is called B800 $(800 \mathrm{~nm})$. The B800-Bchls $a$ are sparsely distributed, while B850-Bchls $a$ are more closely aligned with each other. The EET rate from $\mathrm{B} 800$ to $\mathrm{B} 850$ was determined to be $0.7-0.8$ ps by (a)

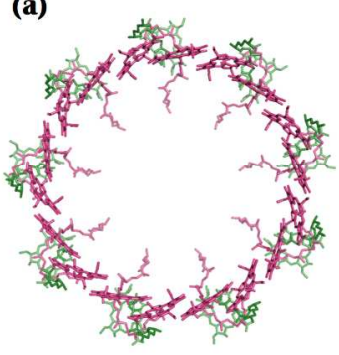

(b)

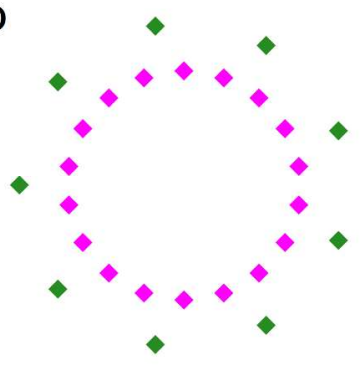

Figure 1. The upper view of LH2 of Rhodopseudomonas acidophila (PDBID: 1NKZ)(2). Only bacteriochlorophylls a (Bchls a) in LH2 are shown for clarity. The inner and outer rings correspond to B850 (magenta) and B800 (green), respectively. (a) The crystal structure from the X-ray structural analysis. (b) The structure in our dipole approximation model.

experimental $(3,4,5,6,7)$ and theoretical $(8,9,10,11$, 12) methods. From the theoretical studies of EET in $\mathrm{B} 850$, it was found that the ring structure possesses unusual properties such as a large dipole moment, large delocalization of excited energy and isotropic nature of excitonic dipoles $(13,14)$. 
Light absorption properties of photosynthesis have attracted considerable attention in astrobiology as a prospective candidate for biosignatures. Presently, more than 1800 extrasolar planets have been confirmed, most of them by the Kepler space telescope (NASA) launched in 2009. In recent years, astronomers have been planning to search for second earths, which are habitable planets in extrasolar systems. In order to find evidence of life in extrasolar planets, the detection of signals from photosynthetic organisms is most promising, because photosynthesis is one of the oldest phenomena in the history of the earth. Nowadays, reflection spectrum of the earth can be measured by remote sensing. The vegetation red edge (VRE) is one characteristic feature of reflection spectra, which can be distinguished from the reflection spectrum of the Earth by the earthshine measurements $(15,16,17,18,19,20,21)$. In the present-day earth, the main contributor of a red edge except for that from higher plants is photosynthetic microbial life, such as bacterial mat and red algae in acid marine drainage (AMD) $(22,23,24)$. However, second earths in extrasolar planetary systems are expected to be planets orbiting M stars, which are cooler than Sun (26). In such conditions, photosynthetic organisms that absorb longer wavelength radiation, e.g., purple bacteria, are likely to be responsible for a red edge. The signature of purple bacteria can indeed be distinguished from the disk-averaged spectra of a present-day Earth with continents covered by deserts, vegetation or microbial mats (25). In addition, the photosynthetic properties of purple bacteria are easier to investigate compared to those of more complex photosynthetic organisms. Thus, exploring how effectively purple bacteria absorb photons in various radiation environments, is a first important step to predict biosignatures on extrasolar planets.

In this study, we have investigated characteristic light absorption and EET of LH2 in purple bacteria. We have constructed models of single-, double- and aggregated LH2 systems to elucidate the intrinsic nature of the light-harvesting mechanism. Given the astrobiological relevance, we simulate efficiencies of light absorption under typical radiation environments for extrasolar planets using the LH2 models.

\section{Computational details}

We have performed static and dynamic calculations using LHC models. In the static calculations, the LHC model is composed of two rings of $\mathrm{BChl}$ a molecules. The transition dipole moment $\mu_{k}$ of each $\mathrm{BChl}$ a pigment was calculated by a time-dependent density functional theory (TDDFT) (27) at the B3LYP/6-31G(d) theoretical (29) level. The Gaussian 09 program package was used for the TDDFT calculations (28). The experimental excited energy $E_{k}$ was used for each pigment, because there are slight differences between the 
experimental and the TDDFT results. $E_{k}$ from experiment and $\mu_{k}$ from TDDFT are used consistently in this paper. Pigment-pigment interactions are approximated by a dipole-dipole interaction to reduce computational cost. Absorption spectra of LHC clusters were calculated by static calculations. A LH2 crystal structure from Rhodopseudomonas acidophila purple bacteria (PDBID: 1NKZ (30)) was used to align the Bchls a in LH2. Dynamical simulations were performed using the same theoretical model.

The Hamiltonian is expressed by the sum of a non-interaction term $H_{0}$ and an interaction term $H_{\text {int }}$.

$$
H_{\text {all }}=H_{0}+H_{\text {int }} \text {. }
$$

$H_{0}$ can be written:

$$
H_{0}=\sum_{k=0}^{N} E_{k} a_{k}^{+} a_{k}
$$

where $E_{k}$ is the transition energy of the $k$-th exciton from the ground state to an excited state. $a_{k}^{+}$and $a_{k}$ are creation and annihilation operators of the $k$-th exciton. The off-diagonal term $H_{\text {int }}$ is approximated by dipole-dipole interactions, which have often been used for LHCs $(31,32,33) . H_{\text {int }}$ is expressed as:

$H_{\text {int }}=\sum_{k<l}^{N} \frac{\mu_{k} \mu_{l}\left\{\cos \left(\theta_{k_{l}}-\theta_{l_{k}}\right)-3 \cos \theta_{k_{l}} \cos \theta_{l_{k}}\right\}}{4 \pi \varepsilon R_{k l}^{3}} a_{k}^{+} a_{l}$,

where $\mu_{k}$ is the transition dipole moment of the $k$-th exciton from the ground state to an excited state, $R_{k l}$ is the distance between the $k$-th and the $l$-th excitons, $\theta_{k_{l}}$ is the angle between the $k$-th and the $l$-th excitons, and $\varepsilon=\kappa \varepsilon_{0}$ is the dielectric constant. $\kappa$ is the effective dielectric constant, and $\varepsilon_{0}$ is the dielectric constant of vacuum. We used $\kappa=3.0(34,35,36)$.

For quantum dynamics simulations, we solved the Liouville equation (37):

$$
i \hbar \frac{\partial}{\partial t} \rho(t)=[H(t), \rho(t)]-i \Gamma \rho(t),
$$

where $\rho(t)$ is the density matrix operator at time $t$, $\hbar=h / 2 \pi$ and $h$ is Planck's constant. The second term on the right-hand side is the relaxation term.

Assuming that the incident light reaches the system, the time-dependent Hamiltonian is given as below. A time-dependent external field term $V(t)$ is added to the Hamiltonian $H_{\text {all }}^{\prime}$ obtained by solving the eigenvalue problem with respect to $H_{\text {all }}$.

$$
\begin{aligned}
H(t) & =H_{a l l}^{\prime}+V(t) \\
& =\sum_{m}^{M} E_{m}^{\prime} b_{m}^{+} b_{m}-\sum_{m, n}^{M} \mu_{m n}^{\prime}(F \cos \omega t) b_{m}^{+} b_{n} .
\end{aligned}
$$

In this study, we applied no external fields. The excitation of an eigenstate or a site, and the subsequent time-evolution are alternately determined.

In the system with $M$ eigenstates, $E_{m}^{\prime}$ is the transition energy in the $m$-th eigenstate, and $\mu_{m n}^{\prime}$ is the transition dipole moment from the nth to the $m$-th 
eigenstate. $b_{m}^{+}$and $b_{m}$ are creation and annihilation operators in the $m$-th state. $F$ is the electric field intensity, and $\omega$ is the frequency of the external field.

The integration of Eq. (4) was accomplished by the 4th order Runge-Kutta method. The time step for integration is $0.001 \mathrm{ps}$ to reproduce reasonable exponential increase or decrease of the density.

We introduce a relaxation term in order to include effects from outside the system. The formulae are expressed as follows. The population relaxation is:

$$
\{-\Gamma \rho(t)\}_{m n}=-\Gamma_{m m} \rho_{m m}+\sum_{n \neq m}^{N} \gamma_{n m} \rho_{n n} .
$$

The coherent relaxation is:

$$
\{-\Gamma \rho(t)\}_{m m}=-\Gamma_{m n} \rho_{m n} \quad m \neq n .
$$

Here,

$$
\begin{gathered}
\Gamma_{m m}=\sum_{n \neq m}^{N} \gamma_{m n}, \\
\Gamma_{m n}=\frac{1}{2}\left(\Gamma_{m m}+\Gamma_{n n}\right)+\Gamma_{m n}^{\prime}, \\
\Gamma_{m n}=\Gamma_{n m},
\end{gathered}
$$

where $\Gamma_{m n}^{\prime}$ is the pure dephasing factor that arises from phase-changing collisions. In a closed system, $\Gamma_{m n}^{\prime}=0$.

We set the feeding parameter $\gamma_{m n}$ as

$$
\gamma_{m n}=C\left(\mathrm{E}_{n}^{\prime}+\mathrm{E}_{m}^{\prime}\right) \quad m<n .
$$

$C$ is a positive real number. In this work, transitions to the ground state $E_{0}$ are prohibited. Relaxation parameters in the relaxation terms were evaluated for the LHC monomer and dimer models. 


\section{Results}

We performed TDDFT calculations on Bchls a in a single LH2. One LH2 contains 27 Bchls a (Figure 1), where 18 Bchls a belong to $\mathrm{B} 850$, and 9 Bchls to $\mathrm{B} 800$. One LH2 has a nine-fold rotational symmetry in the pigment arrangement and the unit is composed of $3 \mathrm{Bchls}$ a. Therefore we performed calculations for a unit of three Bchls. The calculated magnitudes of the transition dipole moment for the 1st excited state are $2.76,2.73$ and 2.73 au for 2 Bchls a in B850 and 1 Bchl a in B800, respectively. These vectors were converted to the LH2 system. The experimental excitation energies for the 1 st excited state, the $\mathrm{Q}_{\mathrm{y}}$ are $1.46 \mathrm{eV}(850 \mathrm{~nm})$ and $1.55 \mathrm{eV}$ $(800 \mathrm{~nm})$ for pigments of $\mathrm{B} 850$ and $\mathrm{B} 800$, respectively. In our theoretical model, calculated transition dipole moments and experimental transition energies for only the 1st excited state of each pigment were used, because that excitation state plays the main role in the energy transfer. As shown in Figure 2, oscillator strength for one LH2 model composed of $27 \mathrm{BChls}$ a has a maximum at 804.67 and $890.62 \mathrm{~nm}$. These two values correspond to maxima for B800 and B850, respectively. By forming the BChls-aggregation in the LH2 systems, redshifts are observed especially at longer wavelengths. These values are in good agreement with the corresponding experimental data at about 800 and $860 \mathrm{~nm}$ (see Figure 1. from 38).

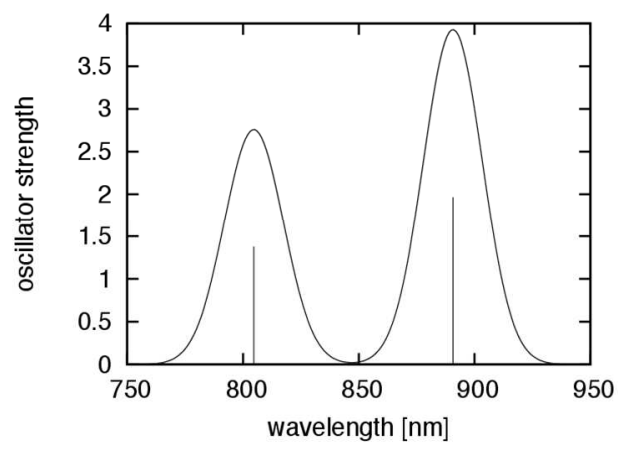

Figure 2. The oscillator strength for one LH2 model composed of 27 BChls a, whose 1st excited state is only considered for each BChls a separately. The absorption lines of the LH2 are broadened by a Gaussian shape function with half-band width of $30 \mathrm{~nm}$.

The oscillator strength at $804.67 \mathrm{~nm}$ belongs to the degenerate 2 nd and 3 rd excited states, while that at $890.62 \mathrm{~nm}$ belongs to the degenerate 20th and $21 \mathrm{st}$ excited states. As shown in Figure 3, the exciton distributions of the 2nd and 3rd states are mainly populated over the B800 sites, and their total transition dipole moments are perpendicular to each other. On the other hand, the 20th and 21st states are mainly populated over the B850 sites, and their total transition dipole moments are also perpendicular to each other. In the 1 st excited state, the density distribution in B850 is almost uniform, and the total dipole moment is almost zero, i.e., direct photo-excitation does not occur between the ground state and the 1st excited state.

In order to determine the relaxation parameters $C$ and $\Gamma^{\prime}$, dynamics simulations were performed using a single LH2 model. We selected the 20th excited state $(804.67$ 
$\mathrm{nm})$ to measure the energy transfer from B800 to B850 sites, because the 20th excited state has a large transition dipole moment and is the lowest excited state distribution in B800. The time-dependence of the density for the 20th excited state is shown in Figure 4. In order to reproduce the experimental energy transfer time of 0.8 ps $(3,4,5,6$, $7,12)$, values of $C=0.545$ and $\Gamma^{\prime}=0.01$ are determined. Henceforth, we used these values for the relaxation parameters.

(a)

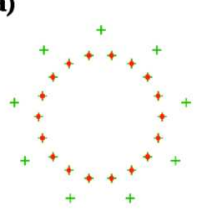

(d)

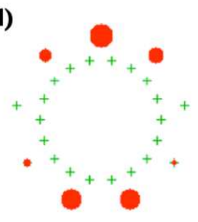

(e)

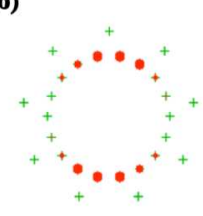

(c)
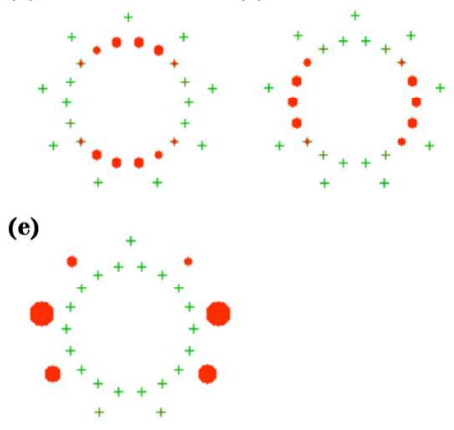

Figure 3. The exciton distributions of key excited states. Green crosses represent the positions of BChls, and the radius of red circles represents the magnitude of each density. (a) The 1st excited state at $940.05 \mathrm{~nm}$, (b) The 2nd excited state at $890.62 \mathrm{~nm}$, (c) The 3rd excited state at $890.62 \mathrm{~nm},(\mathrm{~d})$ The 20th excited state at $804.67 \mathrm{~nm}$, and (e) The 21st excited state at $804.67 \mathrm{~nm}$. The $2 \mathrm{nd}$ and 3rd excited states are degenerate and the total transition dipole moments are perpendicular to each other. This also applies to the 20th and 21st excited states, although they are mainly populated over the outer B800 ring.

Dynamics simulations from several excited states were performed up to $100 \mathrm{ps}$. For example, in one simulation we started from the 3 rd excited state, and as the population of the $3 \mathrm{rd}$ excited state decreased, the population of the 1 st excited state increased without exciting the 2nd state (Figure 5 (a)). In another simulation started from the $21 \mathrm{st}$ state, the $21 \mathrm{st}$ state decayed rapidly within 10 ps and the population of the 1st excited state increased through the 2nd and 3rd excited states. It is noted that the population of the 20th excited state is unchanged (Figure 5 (b)). For both simulations, the final state is always the first excited state. This result is expected because the EET to the ground state was prohibited in our model. This behavior is justified as long as we are considering picosecond time scales.

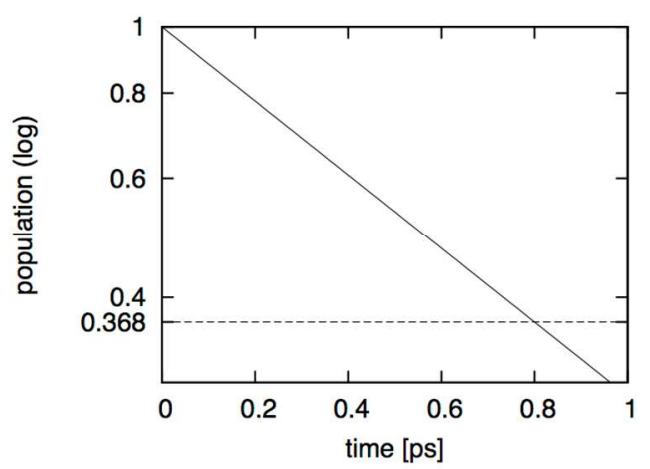

Figure 4. The time evolution of the density of the 20th excited state calculated in single LH2. Linear decay (single exponential decay) was clearly observed in the initial relaxation process. 
Figure 5. Relaxation dynamics from pure excited states. Populations of the 1st, 2nd and 21 st excited states are shown as black, magenta and blue curves, respectively. (a) The 3rd excited state was initially excited.

(b) The 21 st excited state was initially excited. The density of the 2 nd state (not shown in the figure) follows that of the $3 \mathrm{rd}$ excited state very closely.

Energy transfer calculations were performed using two LH2s. The distance between two LH2s was set to $2.7 \mathrm{~nm}$ based on experimental results of 1.5-3.8 nm observed with atomic force microscopy $(39,40,41)$. In order to investigate the EET between the LH2s, the initial state chosen was one for which one site in an inner ring (B850) in ring1 was excited. Figure 6 shows the exciton population for each ring. As the sum of the exciton

populations in ring1 is damping slowly, the exciton population in ring2 is increasing. From the population decrease in ring 1, the EET time constant was determined to $1.33 \mathrm{ps}$. The calculated result is in good agreement with the experimentally determined values of 2-10 ps (31, 42). Therefore, we deem our simulation is good enough to discuss the dynamical behavior in LH2s.

In in-vivo situations, LH2s are aligned on a triangle lattice in membranes. One LH2 is surrounded by $6 \mathrm{LH} 2 \mathrm{~s}$ for the first shell and $12 \mathrm{LH} 2 \mathrm{~s}$ for the second shell. We therefore constructed more realistic systems, composed of $7 \mathrm{LH} 2 \mathrm{~s}$ and $19 \mathrm{LH} 2 \mathrm{~s}$, which we refer to as $7 \mathrm{LH}$ and $19 L H$ models, respectively. It is noted that light energy harvested by LH2s are transferred to special pair BChls a in the center of RC-LH1 in purple bacteria. Although the detailed conformation is different, both LH2 and RC-LH1 have ring conformations of BChls a. Here, exciton energy in the central two BChls is exchanged at $870 \mathrm{~nm}$ (referred as red BChls a) in order to implicitly include the BChl a special pair in this theoretical model, rather than using the configuration of RC-LH1 explicitly. We performed two simulation, one that considers the red BChls a, and one that does not. The alignment of LH2s in the $19 L H$ was shown in Figure 7. Just as in the simulation using two LH2s, the distance between LH2s was set to $2.7 \mathrm{~nm}$. One site in B800 in the outer side was initially excited. 


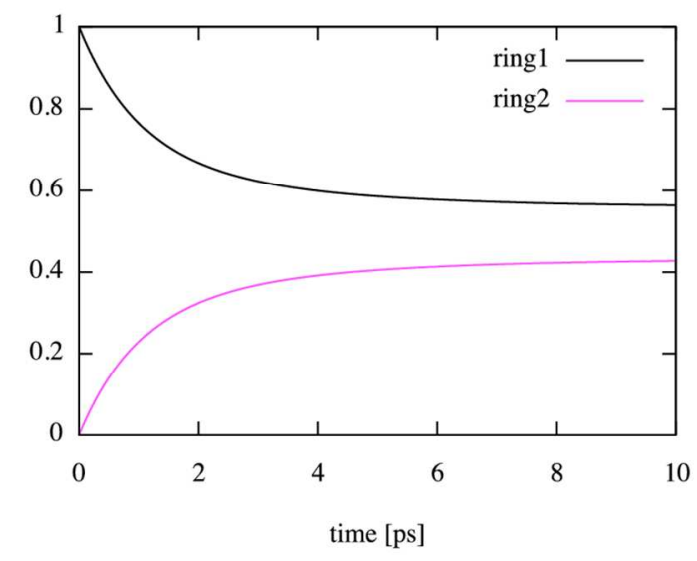

Figure 6. Exciton dynamics in two LH2s. One site in B850 in one LH2 (ring1) is excited in the initial state. The exciton population for each LH2 is shown. The black (magenta) curve corresponds to the sum of densities in ring1 (ring2).

The exciton dynamics simulation of $19 L H$ without red BChls (referred to as $N$ system) showed that the exciton densities are distributed over the B850 almost uniformly after 10 ps (Figure 7 (a)). On the other hand, for a mixed $19 L H$ model (referred to as $R$ system), exciton densities are more localized to the central red BChls after 10 ps. Figure 8 shows the exciton population of $7 L H$ (a) and $19 L H$ (b) models. Rapid EET occurs at an early stage within $0.5 \mathrm{ps}$ and late EET occurs after a time scale of 1 ps. Remarkably, late EET is observed for the mixed system $(R)$. In both the cases of $7 L H$ and $19 L H$, exciton densities of the central BChls in $R$ are almost twice those in $N$. From the early population increases, velocities for long-range energy transfer (v) were evaluated. The distances from the initially excited site in the outer LH2 to the central BChls are 12.07 and $20.80 \mathrm{~nm}$, in $7 \mathrm{LH}$ and
$19 L H$ systems, respectively. Energy transfer velocities were calculated to be $\mathrm{v} /\left(\mathrm{nm} \mathrm{ps}^{-1}\right)=96,174,368,727$ for $7 L H(N), 7 L H(R), 19 L H(N)$ and $19 L H(R)$, respectively (Table 1). These results clearly show that energy transfer in a larger system is much faster than in a smaller system. It is important to mention that energy transfer is accelerated by a factor of two by changing the two central pigment excitation energy. (a)

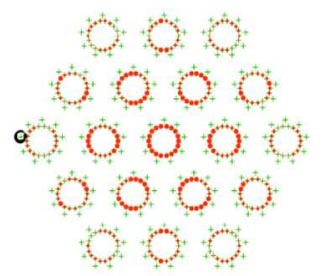

(b)

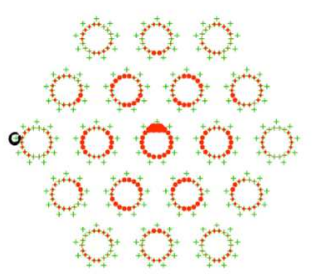

Figure 7. Exciton population on the 19 LH2 models. Green crosses represent the BChl pigments. Exciton populations after 10 ps are shown. One site of B800 marked in a black circle was excited. (a) No red BChls are included in this model $(N)$. (b) The central two BChls are replaced with red BChls with excitation energy at $870 \mathrm{~nm}(R)$.

Absorption spectra for (a) $7 L H$ and (b) $19 L H$ models are shown in Figure 9. Compared to the results in single LH2 (Figure 2), absorption bands are redshifted especially at longer wavelengths (in the region above 875 $\mathrm{nm})$. As the aggregation of LH2 increases, the bands are more redshifted. The calculated absorption peaks are 
$\square / \mathrm{nm}=910$ and 922 for $7 L H$ and $19 L H$, compared to $\square / \mathrm{nm}=891$ for a single LH2. One absorption band observed in the $850-950 \mathrm{~nm}$ region for single LH2 and $7 \mathrm{LHs}$ was splitted into two bands in $19 \mathrm{LH}$ s.

Table 1. The transfer velocities in $7 L H$ and $19 L H$ systems. In each case, systems with two pigments in the central antenna exchanged with two red Bchls $(870 \mathrm{~nm})$, as well as simulations without the exchange, were calculated. The unit is $\mathrm{nm} / \mathrm{ps}$.

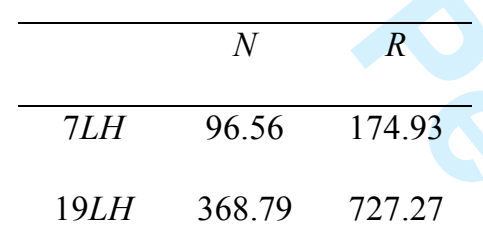

(a)

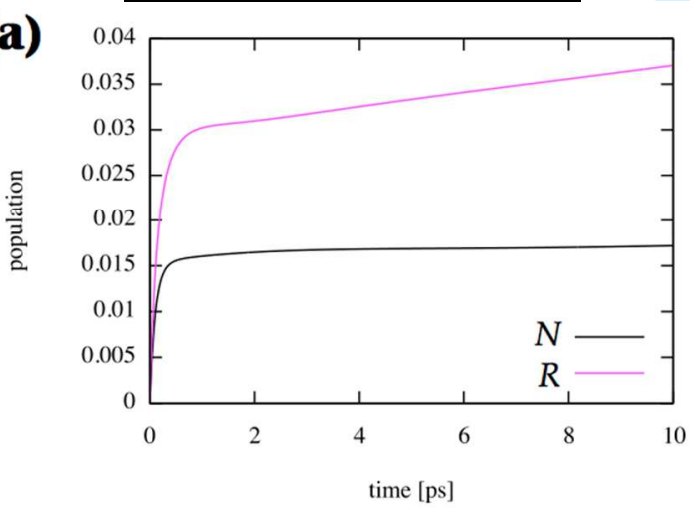

(b)

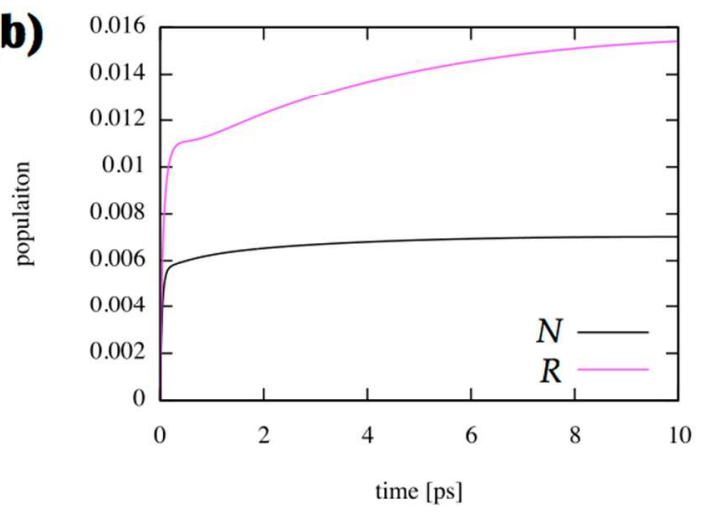

Figure 8. The exciton populations of the central two BChls for (a) $7 \mathrm{LH}$ and (b) $19 L H$ systems. Black and magenta curves are results for $N$ and $R$ systems, respectively.

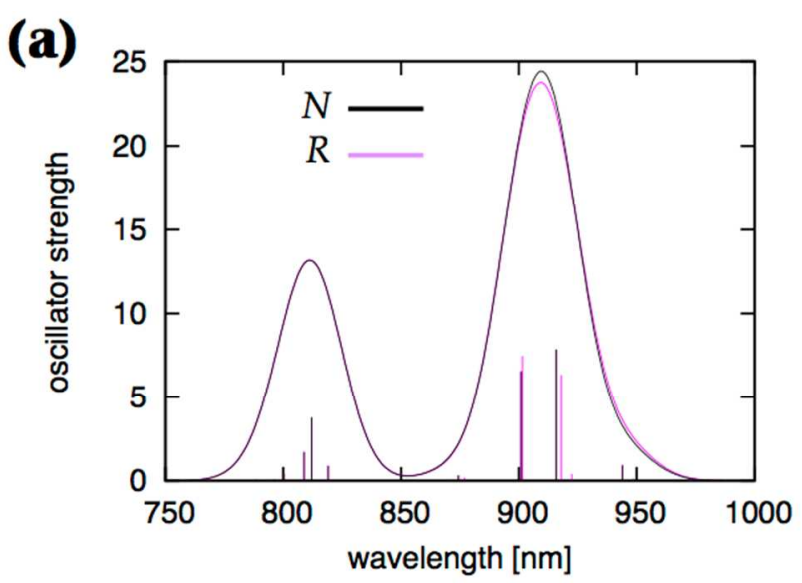

(b)

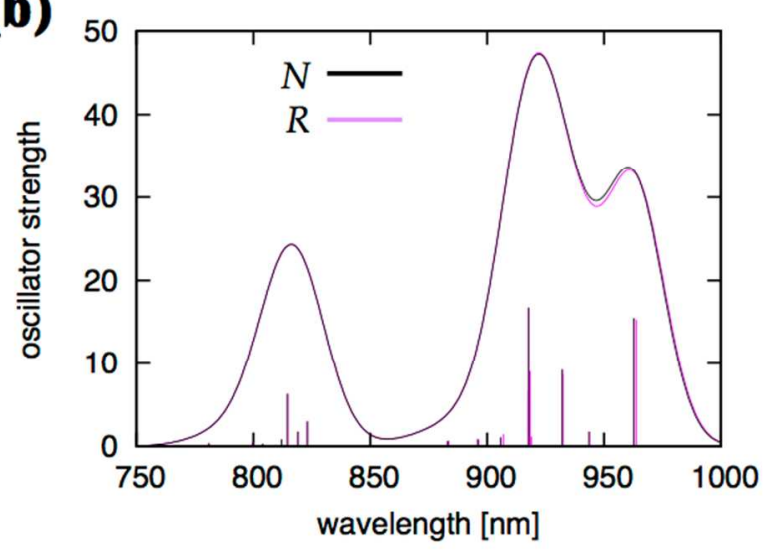

Figure 9. Absorption spectra for (a) $7 L H$ and (b) $19 L H$.

\section{Discussion}

In order to examine the light absorption efficiencies for LH2 antenna systems exposed to stellar radiation, the absorption efficiencies $\chi(T)$ are calculated with the following equation:

$$
\chi(T) \equiv \frac{\int \varepsilon(\lambda) n(\lambda, T) d \lambda}{\int n(\lambda, T) d \lambda}
$$

where $\varepsilon(\lambda)$ is the absorption strength of LH2 systems at the wavelength $\lambda . n(\lambda, T)$ is the number density of 
photons at $\lambda$ of stellar radiation from a star with effective temperature $T$. In the equation, a photon description, not an energy description, was adequate for a photon transduction process such as photosynthesis $(43,44)$. The pigments and the LH2 aggregation have three major absorption bands, the $\mathrm{Q}_{\mathrm{y}}$, the $\mathrm{Q}_{\mathrm{x}}$ and Soret bands, in order of increasing energies. We calculated higher excited states up to $323 \mathrm{~nm}$ using the same procedure adapted for the Qy band spectra (Figure 10 (a)). That is, transition dipole moments of $\mathrm{BChls}$ were calculated by TDDFT, and experimentally determined values in a diethyl ether solution $(2.16 \mathrm{eV}(574.2 \mathrm{~nm})$ and $3.47 \mathrm{eV}$ (357.6 nm) for the $\mathrm{Q}_{\mathrm{X}}$ and Soret band) were used for the excitation energies. For the $\mathrm{Q}_{\mathrm{y}}$ band spectra, the absorption spectrum is the same as the black curve in Figure 9 (b). The integration of $\lambda$ in Eq. (10) was performed in the 200 to $10,000 \mathrm{~nm}$ region.

We evaluated the efficiencies using real radiation spectra of seven stars: HD128167 (F2V star), HD114710 (G0V low-activity star), HD206860 (G0V high-activity star), the Sun (G2V star), HD22049 (K2V star), GJ644 (M3Ve star) and AD Leo (M4.5V star) $(45,46,47)$. The normalized spectra are shown in Figure 10 (a) together with the LH2 absorption spectrum. The absorption efficiencies of the LH2 system (19LH model) with respect to black body spectra for an effective temperature $T$ are shown in Figure 10 (b), together with the stellar radiation spectra. $\chi(T)$ peaks at the emission spectrum of a black body at $9766 \mathrm{~K}$, offset from the effective temperature of Sun $(5778 \mathrm{~K})$. The efficiency for solar conditions is 0.78 times the maximum efficiency $(\chi(5778 K) / \chi(9766 K)=0.78)$. It should be noted for the seven stellar radiation spectra used in our calculations that these fluxes were acquired at the top of the atmosphere of a planet in the habitable zone (HZ). The $\mathrm{HZ}$ is the region where planets can sustain liquid water, which is essential for life. For cooler stars, the $\mathrm{HZ}$ is closer to the star. Stars are generally categorized into several spectral types. The seven stars used here belong to the F, G, K, M star in order of decreasing effective temperature. Our Sun is categorized as a G2V star. In Figure 10 (b), we see that the efficiencies with respect to stellar radiation are similar to those for black body radiation except for a M3Ve star. Although the black body approximation becomes worse in cooler stars, the difference between a black body and the real stellar radiation is unexpectedly small especially in $\mathrm{M} 4.5 \mathrm{~V}$ star. This is because the fluxes of the black body and stars match well in the longer wavelength region of the $\mathrm{Q}_{\mathrm{y}}$ band. On earth, sunlight is reduced by the atmosphere and geographic factors such as water and soil. In our current approach, these effects are not considered but may influence the efficiencies significantly. As a first step, we have performed a systematic investigation of the direct influence of the primary star. 
(a)

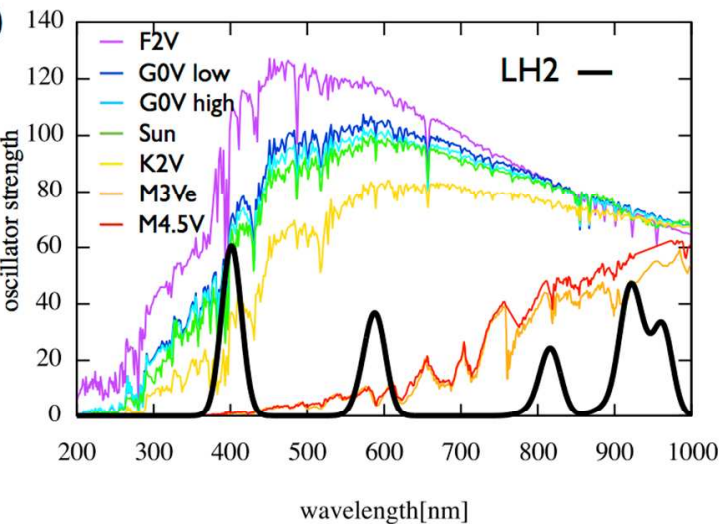

(b)

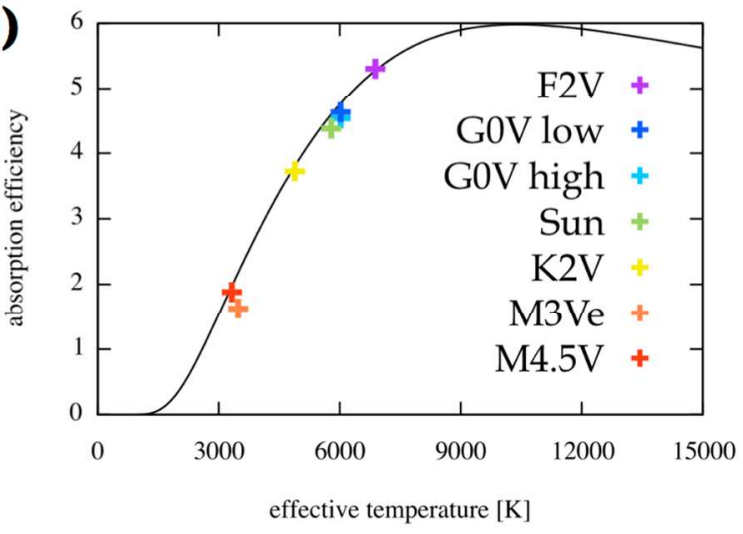

Figure 10. (a) The absorption strength of the $19 L H$ system calculated using the major three excited states of the pigments (black curve). The normalized stellar fluxes are also shown: F2V, G0V low-activity, G0V high-activity, K2V, M4.5V and M3Ve stars and the Sun. The flux of the Sun is normalized at maximum to 100.0. The other fluxes are normalized so as to show the same integrated flux from 200 to 10,000 $\mathrm{nm}$ to the normalized flux of the Sun. (b) Absorption efficiencies calculated using the seven stellar radiation spectra (plotted as cross marks) and the blackbody spectrum (black curve). The efficiency is maximized at the emission spectrum of a black body at $9766 \mathrm{~K}$.

\section{Conclusions}

The light absorption spectra and energy transfer mechanism of LH2 systems in purple bacteria were investigated through quantum dynamics simulations. We determined the relaxation parameters using small LH2 models, single LH2 and double LH2 models. The LH2 models reproduced appropriate values found in experiments: $0.8 \mathrm{ps}$ for the B800-B850 electron transfer and $1.33 \mathrm{ps}$ for the inter-LH2 electron transfer. Then, we constructed aggregate LH2s models, $7 L H$ and $19 L H$. From our calculations, we found that the LH2 aggregate system collects the absorbed excitation energy in the central BChls within a time scale of 1.0 ps. We also found that absorption spectra are red-shifted in LH2 aggregates and the energy transfer becomes faster. Interestingly, by exchanging the central $\mathrm{BChls}$ to red BChls, the energy transfer velocity increased significantly and the exciton was more localized at the central BChls. Using the LH2 aggregate models, we have investigated the absorption efficiencies with respect to spectra of stars of different stellar types. Both black body and realistic stellar radiation spectra were examined. We found that the absorption efficiency was maximized at $9766 \mathrm{~K}$ and the black body approximation was valid for higher temperature stars. For cooler stars like M stars, the black body approximation becomes worse, and real stellar spectra should be used.

In future, when more detailed and realistic photo-environments are investigated, influences from the atmosphere, geometric contributions, and the structure of vegetation must be taken into account as the may 
strongly affect absorption efficiencies. Therefore, real stellar spectra and planet environments need to be considered to evaluate absorption efficiencies.

In order to detect biosignatures from extrasolar planets, the prediction of the efficiency of photosynthetic systems in extrasolar planets must be made. To this end, a systematic approach evaluating the photosystems from the electronic level to large macroscopic levels must be adopted. In this study, mechanisms of the light absorption and energy transfer in aggregate LH2 systems were investigated. This work is a first important step to construct more sophisticated theoretical models valid not only for the photosystems on earth but also for photosystems in extrasolar planets.

\section{Acknowledgements}

This work was supported by Grant-in-Aid for JSPS Fellows (Y.K. 26.1303) from Japan Society for the Promotion of Science (JSPS).

\section{Supplemental data}

Supplemental data for this article can be accessed here.

\section{References}

[1] G. McDermott, S.M. Prince, A.A. Freer, A.M. Hawthornthwaite-Lawless, M.Z. Papiz, R.J. Cogdell, N. W. Isaacs, Science 374, 517 (1995).
[2] A.M. van Oijen, M. Ketelaars, J. Köhler, T.J. Aartsma, J. Schmidt, Science 285, 400 (1999).

[3] J.L. Herek, W. Wohlleben, R.J. Cogdell, D. Zeidler, M. Motzkus, Nature 417, 533 (2002).

[4] J.A. Ihalainen, J. Linnanto, P. Myllyperkiö, I.H.M. van Stikkum, B. Ücker, H. Scheer, J.E.I. Korppi-Tommola, J. Phys. Chem. B 105, 9849 (2001).

[5] C.D. Caro, R.W. Visschers, R. van Grondelle, S. Völker, J. Phys. Chem. 98, 10584 (1994).

[6] F.G. Zhang, R. van Grondelle, V. Sundström, Biophys. J. 61, 911 (1992).

[7] A. Freiberg, J.P. Allen, J.C. Wiliams, N.W. Woodbury, Photosynth. Res. 48, 309 (1996)

[8] H. Sumi, J. of Luminescence 87-89, 71 (2000).

[9] H. Sumi, J. Phys. Chem. B 103, 252 (1999).

[10] G.D. Scholes, G. R. Fleming, J. Phys. Chem. B 104, 1854 (2000).

[11] R. Jimenez, S.N. Dikshit, S.E. Bradforth, G.R. Fleming, J. Phys. Chem 100, 6825 (1996).

[12] Y.Z. Ma, R.J. Cogdell, T.J.; Gillbro, Phys. Chem. B 101, 1087 (1997).

[13] M. Nakatani, G. Tei, H. Ishihara, Phys. Status Solidi B 248, 448 (2011).

[14] G. Tei, M. Nakatani, H. Ishihara, Phys. Status Solidi B 248, 399 (2011).

[15] N.Y. Kiang, J. Siefert, Govindjee, R.E. Blankenship, Astrobiology 7, 222 (2007).

[16] L. Arnold, S. Gillet, O. Lardière, P. Riaud, J. 
Schneider, A\&A 392, 231 (2002).

[17] N.J. Woolf, P.S. Smith, W.A. Traub, K.W. Jucks, APJ 574, 430 (2002).

[18] S. Seager, E.L. Turner, J. Schafer, E.B. Ford, Astrobiology 5, 372 (2005).

[19] P. Montañés-Rodriguez, E. Pallé, P.R. Goode, J. Hickey, S.E. Koonin, APJ 629, 1175 (2005).

[20] G. Tinetti, V.S. Meadows, D. Crisp, W. Fong, E. Fishbein, M. Turnbull, J.-P. Bibring, Astrobiology 6, 34 (2006a).

[21] G. Tinetti, V.S. Meadows, D. Crisp, N.Y. Kiang, B.H. Kahn, E. Bosc, E. Fishbein, T. Velusamy, M. Turnbull, Astrobiology 6, 881 (2006b).

[22] R.F. Kokaly, R.N. Clark, K.E. Livo, in Summaries of the 7th Annual JPL Airborne Earth Science Workshop, JPL Publ. 97-21, edited by Green, R. O. pp. 235-244, Jet Propul. Lab, Pasadena, Calif., 1998.

[23] R.F. Kokalya, D.G. Despainb, R.N. Clarka, K.E. Livo, Remote Sens. Environ. 84, 437 (2003).

[24] R.F. Knacke, Astrobiology 3, 531 (2003).

[25] E. Sanromá, E. Pallé, M.N. Parenteau, N.Y. Kiang, A.M. Gutiérrez-Navarro, R. López, P. Montáñes-Rodríguez, APJ 780, 52 (2014).

[26] J.Scalo, L. Kaltenegger, A. Segura, M. Fridlund, I. Ribas, Y.N. Kulikov, J.L. Grenfell, H. Rauer, P. Odert, M. Leitzinger, F. Selsis, M.L. Khodachenko, C. Eiroa, J. Kasting, H. Lammer, Astrobiology 7, 85 (2007).

[27] M.A.L. Marques, E.K.U. Gross, Annu. Rev. Phys.
Chem. 55, 427 (2004).

[28] M.J. Frisch, G.W. Trucks, H.B. Schlegel, G.E. Scuseria, M.A. Robb, J.R. Cheeseman, G. Scalmani, V. Barone, B. Mennucci, G.A. Petersson, H. Nakatsuji, M. Caricato, X. Li, H.P. Hratchian, A.F. Izmaylov, J. Bloino, G. Zheng, J.L. Sonnenberg, M. Hada, M. Ehara, K. Toyota, R. Fukuda, J. Hasegawa, M. Ishida, T. Nakajima, Y. Honda, O. Kitao, H. Nakai, T. Vreven, J.A. Montgomery, Jr., J.E. Peralta, F. Ogliaro, M. Bearpark, J. J. Heyd, E. Brothers, K.N. Kudin, V.N. Staroverov, R. Kobayashi, J. Normand, K. Raghavachari, A. Rendell, J. C. Burant, S.S. Iyengar, J. Tomasi, M. Cossi, N. Rega, J. M. Millam, M. Klene, J.E. Knox, J. B. Cross, V. Bakken, C. Adamo, J. Jaramillo, R. Gomperts, R.E. Stratmann, O. Yazyev, A.J. Austin, R. Cammi, C. Pomelli, J.W. Ochterski, R.L. Martin, K. Morokuma, V.G. Zakrzewski, G.A. Voth, P.Salvador, J.J. Dannenberg, S. Dapprich, A.D. Daniels, Ö. Farkas, J.B. Foresman, J.V. Ortiz, J. Cioslowski, and D.J. Fox, Gaussian 09, Revision D.01, Gaussian, Inc., Wallingford CT, 2009.

[29] D.A. Becke, Phys, Rev. A 38, 3098 (1988).

[30] M.Z. Papiz, S.M. Prince, T. Howard, R.J. Cogdell, N.W. Isaacs, J. Mol. Biol. 326, 1523 (2003).

[31] J.M. Linnanto, J.E.I. Korppi-Tommola, Chem. Phys. 357, 171 (2009).

[32] J.M. Linnanto, J.E.I. Korppi-Tommola, J. Chin. Chem. Soc. 47, 657 (2000).

[33] J.M. Linnanto, J.E.I. Korppi-Tommola, V.M. 
Helenius, J. Phys. Chem. B 103, 8739 (1999).

[34] J.N. Sturgis, B. Robert, Photosynth. Res. 50, 5 (1996).

[35] K. Sauer, R.J. Cogdell, S.M. Prince, A. Freer, N.W. Isaacs, H. Scheer, Photochem. Photobiol. 64, 564 (1996).

[36] C. Curutchet, J. Kongsted, A. Muñoz-Losa, H. Hossein-Nejad, G.D. Scholes, B.J. Mennucci, Am. Chem. 133, 3078 (2011).

[37] M. Nakano, K. Yamaguchi, Phys. Rev. A 1994, 50, 4, 2989.

[38] R.J. Cogdell, N.W. Isaacs, A.A. Freer, T.D. Howard, A.T. Gardiner, S.M. Prince, M.Z. Papiz, $\square$ FEBS Letters 555, 35 (2003).

[39] S. Scheuring, F. Reiss-Husson, A. Engel, J.-L. Rigaud, J.-L. Ranck, EMBOJ. 20, 3029 (2001).

[40] A. Stamouli, S. Kafi, D.C.G. Klein, T.H. Oosterkamp, J.W.M. Frenken, R. J. Cogdell, T. J. Aartsma, Biophys. J. 84, 2483 (2003).
[41] S. Scheuring, J.N. Sturgis, V. Prima, A. Bernadac, D. Lévy, J.-L. Rigaud, Proc. Nat. Acad. Sci. USA. 101, 11293 (2004).

[42] R. Agarwal, A.H. Rizvi, B.S. Prall, J.D. Olsen, C.N. Hunter, G.R. Fleming, J. Phys. Chem. A 106, 7573 (2002).

[43] M. Chen, R.E. Blankenship, Trends in Plant Science 16, 427 (2011).

[44] R.E. Blankenship, M. Chen. Curr. Opin. Chembiol. 17, 457 (2013).

[45] C. Wehrli, WRC PMOD Pub. No. 615 (1985).

[46] A. Segura, J.F. Kasting, V. Meadows, M. Cohen, J. Scalo, D. Crisp, R.A.H. Butler, G. Tinetti, Astrobiology 5, 706 (2005).

[47] A. Segura, K. Krelove, J.F. Kasting, D. Sommerlatt, V. Meadows, D. Crisp, M. Cohen, E. Mlawer, Astrobiology 3, 689 (2003). 
Supplemental data

\title{
Light absorption and excitation energy transfer calculations in primitive photosynthetic bacteria
}

\author{
Yu Komatsu ${ }^{\mathrm{a}, ~ *}$, Megumi Kayanuma ${ }^{\mathrm{b}}$, Mitsuo Shoji ${ }^{\mathrm{a}}$, Kazuhiro Yabana ${ }^{\mathrm{a}}$, Kenji Shiraishic ${ }^{\mathrm{c}}$ \\ and Masayuki Umemura ${ }^{a}$ \\ ${ }^{a}$ Graduate School of Pure and Applied Sciences, University of Tsukuba, Tsukuba, Japan \\ ${ }^{\mathrm{b}}$ Graduate School of Systems and Information Engineering, University of Tsukuba, \\ Tsukuba, Japan \\ ${ }^{\mathrm{c}}$ Graduate School of Engineering, Nagoya University, Nagoya, Japan
}

\section{Von-Neumann Liouville equation}

Here, the derivation of the general Von-Neumann Liouville equation is represented, as is to be dealt with the time evolution of the density.

The time-dependent density matrix $\rho(t)$ can be represented using the time-dependent wave function $\Psi(t)$.

$$
\rho(t) \equiv|\Psi(t)\rangle\langle\Psi(t)|
$$

And its differential with respect to $t$ is:

$$
\frac{\partial \rho(t)}{\partial t}=\left(\frac{\partial}{\partial t}|\Psi(t)\rangle\right)\langle\Psi(t)|+| \Psi(t)\rangle\left(\frac{\partial}{\partial t}\langle\Psi(t)|\right) .
$$

Time-dependent Schrödinger equation is expressed as follows.

$$
i \hbar \frac{\partial}{\partial t}|\Psi(t)\rangle=H|\Psi(t)\rangle .
$$

And its Hermitian conjugate form is:

$$
-i \hbar \frac{\partial}{\partial t}\langle\Psi(t)|=\langle\Psi(t)| H .
$$

Eq. (3) and (4) are substituted into Eq. (2).

$$
\begin{aligned}
\frac{\partial \rho(t)}{\partial t} & =\left(\frac{1}{i \hbar} H|\Psi(t)\rangle\right)\langle\Psi(t)|+| \Psi(t)\rangle\left(-\frac{1}{i \hbar}\langle\Psi(t)| H\right) \\
& =\frac{1}{i \hbar}(H \rho-\rho H) \\
& =\frac{1}{i \hbar}[H, \rho] .
\end{aligned}
$$

The last column in the equation corresponds to the general representation of Von-Neumann Liouville equation. 


\section{External field and oscillator strength}

The electric field term is induced to the Hamiltonian as follows. In our dipole-dipole approximation, interactions between the electric dipole moment $\boldsymbol{p}$ and the electric field $\boldsymbol{E}$ are considered. By using these, the electric field term is represented as

$$
-\boldsymbol{p} \cdot \boldsymbol{E} .
$$

When $\boldsymbol{p}=\left(p_{x}, p_{y}, p_{z}\right)$, the electric field along $\mathrm{x}$ axis is added as $\boldsymbol{E}=(F \cos \omega t, 0,0)$. The term is to be expressed as

$$
-p_{x}(F \cos \omega t) .
$$

In this case, the dipole moment only along $\mathrm{x}$ axis needs to be considered.

$$
p_{x}=\sum_{m, n=1}^{M} \mu_{m n}^{\prime} b_{m}^{+} b_{n} .
$$

Therefore, the electric field term is shown as below.

$$
-\sum_{m, n=1}^{M} \mu_{m n}^{\prime}(F \cos \omega t) b_{m}^{+} b_{n} .
$$

The general description of the transition moment from eigenstate $n$ to $m$ is

$$
\begin{aligned}
\mu_{m n}^{\prime} & =\left\langle\psi_{m}|\mu| \psi_{n}\right\rangle \\
& =\sum_{k=0}^{N} \sum_{l=0}^{N}\left\langle\psi_{m} \mid \varphi_{k}\right\rangle\left\langle\varphi_{k}|\mu| \varphi_{l}\right\rangle\left\langle\varphi_{l} \mid \psi_{n}\right\rangle \\
& =\sum_{k=0}^{N} \sum_{l=0}^{N} C_{m k}^{*} C_{n l}\left\langle\varphi_{k}|\mu| \varphi_{l}\right\rangle \\
& =\sum_{k=0}^{N} \sum_{l=0}^{N} C_{m k}^{*} C_{n l} \mu_{k l} .
\end{aligned}
$$

The transition dipole moments in $N$ sites are converted into those from eigenstate $n$ to $m$. Here, we consider single excitation but not multiple excitation to calculate the transition dipole moments. Therefore,

$$
\mu_{m n}^{\prime}=\sum_{k=1}^{N} C_{m k}^{*} C_{n 0} \mu_{k 0}+\sum_{l=1}^{N} C_{m 0}^{*} C_{n l} \mu_{0 l} .
$$

The oscillator strength in the state $m$ is given by

$$
f_{m}=\frac{2 m_{e}}{3 \hbar^{2} e^{2}}\left(E_{m}-E_{0}\right)\left|\mu_{m 0}^{\prime}\right|^{2} .
$$

Subscriptions 0 represent the ground states. $m_{e}$ is the mass of an electron, and $e$ is the charge of an electron. $E_{m}$ is the energy in state $m$.

\section{Relaxation process}

The derivation of the relaxation term is explained. When we focus on a system, all the information of the system is usually not obvious. In the relaxation process, the already-known system which we are interested in is projected on by the other unknown system surrounding the known small system.

As given Hamiltonian $H_{0}$, the eigenstate $|m\rangle$ and the eigenvalue $E_{m}$ have already known here. In that case,

$$
H_{0}|m\rangle=E_{m}|m\rangle \text {. }
$$


To see the contribution of the energy relaxation on a state $m$, Schrödinger equation can be expressed as

$$
\begin{aligned}
& H_{0}^{\prime}|m\rangle=\left(E_{m}+\frac{i \Gamma_{m m}}{2}\right)|m\rangle, \\
& \langle m| H_{0}^{\prime}=\langle m|\left(E_{m}-\frac{i \Gamma_{m m}}{2}\right) .
\end{aligned}
$$

In these equations, the Hamiltonian is given as $H_{0}^{\prime}$. By using $H_{0}^{\prime}$, the time evolution of $|\Psi\rangle$ is expressed as

$$
i \hbar \frac{\partial}{\partial t}|\Psi(t)\rangle=H_{0}^{\prime}|\Psi(t)\rangle
$$

And its solution:

$$
|\Psi(t)\rangle=\sum_{m} a_{m}(0) \exp \left[-\frac{i}{\hbar}\left(E_{m}+\frac{i \Gamma_{m m}}{2}\right) t\right]|m\rangle .
$$

As using this relation, time-dependent population is shown like this:

$$
\begin{aligned}
a_{m}(0) \exp \left[-\frac{i}{\hbar}\left(E_{m}+\frac{i \Gamma_{m m}}{2}\right) t\right] a_{m}^{*}(0) \exp \left[\frac{i}{\hbar}\left(E_{m}-\frac{i \Gamma_{m m}}{2}\right) t\right] & =a_{m}(0) a_{m}^{*}(0) \exp \left[-\frac{\Gamma_{m m}}{\hbar} t\right] \\
& =\rho(0) \exp \left[-\frac{\Gamma_{m m}}{\hbar} t\right] .
\end{aligned}
$$

Therefore, this exhibits exponential damping. With Eq. (19) and (20),

$$
\begin{gathered}
\left\langle m\left|H_{0}^{\prime} \rho(t)\right| m\right\rangle=\left\langle m\left|\left(E_{m}+\frac{i \Gamma_{m m}}{2}\right)\right| \Psi(t)\right\rangle\langle\Psi(t) \mid m\rangle \\
=\left(E_{m}+\frac{i \Gamma_{m m}}{2}\right) \rho_{m m}(t), \\
\left\langle m\left|\rho(t) H_{0}^{\prime}\right| m\right\rangle=\left(E_{m}-\frac{i \Gamma_{m m}}{2}\right) \rho_{m m}(t), \\
\left\langle m\left|H_{0}^{\prime} \rho(t)\right| n\right\rangle=\left(E_{m}+\frac{i \Gamma_{m m}}{2}\right) \rho_{m n}(t), \\
\left\langle m\left|\rho(t) H_{0}^{\prime}\right| n\right\rangle=\left(E_{n}-\frac{i \Gamma_{n n}}{2}\right) \rho_{m n}(t) .
\end{gathered}
$$

By using these equations,

$$
-\frac{i}{\hbar}\left[H_{0}^{\prime}, \rho(t)\right]=\left(\begin{array}{ccc}
-\frac{1}{\hbar} \Gamma_{m m} \rho_{m m}(t) & \cdots & -\frac{1}{\hbar}\left[i\left(E_{m}-E_{n}\right)+\frac{1}{2}\left(\Gamma_{m m}+\Gamma_{n n}\right)\right] \rho_{m n}(t) \\
\vdots & \ddots & \vdots \\
-\frac{1}{\hbar}\left[i\left(E_{n}-E_{m}\right)+\frac{1}{2}\left(\Gamma_{n n}+\Gamma_{m m}\right)\right] \rho_{n m}(t) & \cdots & -\frac{1}{\hbar} \Gamma_{n n} \rho_{n n}(t)
\end{array}\right) .
$$

As considered $H_{0}$,

$$
-\frac{i}{\hbar}\left[H_{0}, \rho(t)\right]=\left(\begin{array}{ccc}
0 & \cdots & -\frac{1}{\hbar}\left[i\left(E_{m}-E_{n}\right)\right] \rho_{m n}(t) \\
\vdots & \ddots & \vdots \\
-\frac{1}{\hbar}\left[i\left(E_{n}-E_{m}\right)\right] \rho_{n m}(t) & \cdots & 0
\end{array}\right) .
$$

By subtracting Eq. (31) from (30),

$$
\left(\begin{array}{ccc}
-\frac{1}{\hbar} \Gamma_{m m} \rho_{m m}(t) & \cdots & -\frac{1}{\hbar}\left[\frac{1}{2}\left(\Gamma_{m m}+\Gamma_{n n}\right)\right] \rho_{m n}(t) \\
\vdots & \ddots & \vdots \\
-\frac{1}{\hbar}\left[\frac{1}{2}\left(\Gamma_{n n}+\Gamma_{m m}\right)\right] \rho_{n m}(t) & \cdots & -\frac{1}{\hbar} \Gamma_{n n} \rho_{n n}(t)
\end{array}\right)
$$


This acts as the energy relaxation. In the case of considering only $H_{0}$,

$$
i \hbar \frac{\partial}{\partial t}|\Psi(t)\rangle=H_{0}|\Psi(t)\rangle
$$

And its solution is:

$$
|\Psi(t)\rangle=\sum_{m} a_{m}(0) \exp \left[-\frac{i}{\hbar} E_{m} t\right]|m\rangle .
$$

Therefore, the density matrix is:

$$
\rho(t)=\left(\begin{array}{ccc}
\rho_{m m}(0) & \cdots & -\frac{1}{\hbar}\left[i\left(E_{m}-E_{n}\right)\right] \rho_{m n}(0) \\
\vdots & \ddots & \vdots \\
-\frac{1}{\hbar}\left[i\left(E_{n}-E_{m}\right)\right] \rho_{n m}(0) & \cdots & \rho_{n n}(0)
\end{array}\right) .
$$

In the above equation, the off-diagonal parts damp gradually. In order to reproduce the behavior, we introduce the phenomenological parameter $\Gamma_{m n}^{\prime}$ to Equation (32).

$$
\left(\begin{array}{ccc}
-\frac{1}{\hbar} \Gamma_{m m} \rho_{m m}(t) & \cdots & -\frac{1}{\hbar}\left[\Gamma_{m n}^{\prime}+\frac{1}{2}\left(\Gamma_{m m}+\Gamma_{n n}\right)\right] \rho_{m n}(t) \\
\vdots & \ddots & \vdots \\
-\frac{1}{\hbar}\left[\Gamma_{n m}^{\prime}+\frac{1}{2}\left(\Gamma_{n n}+\Gamma_{m m}\right)\right] \rho_{n m}(t) & \cdots & -\frac{1}{\hbar} \Gamma_{n n} \rho_{n n}(t)
\end{array}\right)
$$

$\Gamma_{m n}^{\prime}$ is the pure dephasing factor. Here,

$$
\begin{gathered}
\Gamma_{m n} \equiv \frac{1}{2}\left(\Gamma_{m m}+\Gamma_{n n}\right)+\Gamma_{m n}^{\prime}, \\
\Gamma_{m n}=\Gamma_{n m} .
\end{gathered}
$$

As the population in a state decreases, that in the other states increases. By introducing the feeding parameter $\gamma_{m n}$, the relaxation term is expressed as

$$
-\Gamma \rho(t)=\left(\begin{array}{ccc}
-\Gamma_{m m} \rho_{m m}(t)+\sum_{n \neq m} \gamma_{n m} \rho_{n n} & \cdots & -\left[\Gamma_{m n}^{\prime}+\frac{1}{2}\left(\Gamma_{m m}+\Gamma_{n n}\right)\right] \rho_{m n}(t) \\
\vdots & \ddots & \vdots \\
-\left[\Gamma_{n m}^{\prime}+\frac{1}{2}\left(\Gamma_{n n}+\Gamma_{m m}\right)\right] \rho_{n m}(t) & \cdots & -\Gamma_{n n} \rho_{n n}(t)+\sum_{m \neq n} \gamma_{m n} \rho_{m m}
\end{array}\right),
$$

Since the density matrix is Hermit and the sum of population must be conserved, the diagonal parts are added to be zero.

$$
\begin{gathered}
\cdots+\left(-\Gamma_{m m} \rho_{m m}(t)+\sum_{n \neq m} \gamma_{n m} \rho_{n n}\right)+\cdots+\left(-\Gamma_{n n} \rho_{n n}(t)+\sum_{m \neq n} \gamma_{m n} \rho_{m m}\right)=0, \\
\cdots+\left(-\Gamma_{m m}+\sum_{n \neq m} \gamma_{m n}\right) \rho_{m m}(t)+\cdots+\left(-\Gamma_{n n}+\sum_{m \neq n} \gamma_{n m}\right) \rho_{n n}(t)=0 .
\end{gathered}
$$

Therefore, below relation can be obtained.

$$
\Gamma_{m m}=\sum_{n \neq m}^{N} \gamma_{m n}
$$

